\title{
Optical design for an adaptive anastigmatic five-mirror extremely large telescope
}

\author{
B. Delabre \\ European Southern Observatory, Karl-Schwarzschild-Str. 2, Garching, Germany \\ e-mail: bdelabre@eso.org \\ Received 22 August 2007 / Accepted 15 December 2007

\begin{abstract}
Observations in extremely large telescopes will rely on adaptive corrections. To reduce the size and complexity of the instruments observing over large fields, the telescope should correct not only fast telescope misalignments and guiding errors, but also the bulk of the errors introduced by the atmosphere. This paper describes an optical design with five mirrors, three of them powered, which fulfils this requirement. The optical system is effectively diffraction limited over a field of 10 arcmin, also delivering very good image quality of laser guide stars. In addition, it offers the possibility of installing a large field atmospheric dispersion corrector and the freedom to switch between a large number of foci by changing the focal length. A weak field curvature concentric to the exit pupil eases the design of instrumentation.
\end{abstract}

Key words. telescopes

\section{Introduction}

The Ritchey-Chrétien solution has dominated the design of large optical telescopes for many decades. For a two-mirror telescope it is the natural solution for optimizing the image quality over fields of view with diameters of the order of 30 arcmin (Wilson 2004). With the ongoing development of adaptive optics for the correction of wavefront aberrations generated by atmospheric turbulence (Tyson 1998), the requirements on the optical quality of telescopes are increasing and the designs may have to be adapted accordingly. The major design decision will be whether the telescope should supply at least partial adaptive optics corrections, including fast tip-tilt corrections, also known as field stabilisation. If this is the case, there are several possibilities as to how this can be realised by a specific optical design, which will also depend on additional requirements imposed on the telescope.

An overview of several optical designs for telescopes with more than two powered mirrors has been given by Wilson (Wilson 2004). The optical design presented in this paper is based on three-mirror anastigmatic designs (Korsch 1972). It is currently the baseline for the European Extremely Large Telescope (E-ELT) equipped with a primary mirror with approximately 1000 segments having diameters of $1.4 \mathrm{~m}$ corner to corner (Gilmozzi \& Spyromilio 2007). Since the design introduced in this paper has five mirrors, the shorthand notation $\mathrm{M} i$ will be used for the $i$ th mirror in the optical train.

Section 2 contains a list of requirements for an adaptive and instrument-friendly extremely large telescope. Section 3 starts with discussions on the adequacy of optical solutions like a Gregorian or Ritchey-Chretien design and the minimum number of mirrors required for fulfilling the requirements. It continues with a trade-off between a spherical and an aspherical primary mirror. Finally, the five-mirror design is presented, discussing in detail the image quality, the incorporation of adaptive optics, the feasibility of introducing a large-field atmospheric dispersion corrector, and the available focus positions. Throughout the whole paper, the focus will remain on the optical design.

\section{Requirements on the telescope}

The optical design for a telescope with a diameter of the primary mirror of $42 \mathrm{~m}$, presented in this paper, is based on the following list of requirements that can be grouped into two categories. The first category addresses the adaptive optics corrections and the second one, instrument requirements.

A The telescope should be able to correct, at least partially, the wavefront aberrations generated by atmospheric turbulence in the ground layer over a field of view with a diameter of approximately 10 arcmin. Such a correction could also be achieved by a post-focal system, but this would have to be duplicated for all foci and would deliver only a limited field of view of approximately 2 arcmin.

The requirement of adaptive optics corrections in the telescope has the following consequences:

A1 the deformable adaptive mirror should be conjugated to a layer approximately $200 \mathrm{~m}$ above the ground;

A2 to guarantee that all parts of the adaptive mirror are conjugated to approximately the same height, the mirror should not be strongly tilted with respect to the optical axis;

A3 for technological reasons, the adaptive mirror, which corrects the fast shape and residual tilt wavefront errors requiring small strokes, should preferably be flat. Its diameter should not be larger than $2.5 \mathrm{~m}$, but large enough to accommodate a sufficient number of actuators;

A4 since the large strokes required for correcting tilt wavefront errors are difficult to achieve with thin deformable mirrors like already existing adaptive secondaries, the 
correction of the bulk of the fast tip-tilt wavefront errors requiring large strokes should be done by a mirror other than the thin deformable mirror. To avoid strong pupil movements in the instruments, such a mirror should be close to a telescope pupil;

A5 a sufficient sky coverage requires the use of laser guide stars for the adaptive optics system. The wavefront aberrations caused by the finite distance of the laser spots from the telescope should not be significantly larger than the wavefront aberrations generated by atmospheric turbulence.

B The telescope should be instrument-friendly. This can be achieved by the following more specific requirements.

B1 the focal ratio of the telescope should be of the order of 15, which appears to be a good compromise between the size of the field and the complexity of the instrumentation;

B2 the field curvature should be weak and, ideally, concentric to the exit pupil;

B3 the telescope should have a Nasmyth focus, since the expected complex and heavy instruments are best supported on a stable platform;

B4 a gravity stable focus, where the derotation of the field is done by a rotation of the instrument around a vertical axis, would be beneficial for particularly complex instruments;

B5 for instruments with extreme stability requirements, both in terms of gravity and temperature, the telescope should also offer a coudé focus;

B6 the telescope should offer the possibility of correcting for atmospheric dispersion over the full field of view.

\section{Optical design}

\subsection{Is a Gregorian or a Ritchey-Chrétien telescope adequate?}

The choice of a Gregorian or a Ritchey-Chrétien design would have the following consequences:

- the ground layer correction as well as the field stabilization would have to be done by the secondary mirror;

- the size of the secondary mirror would be more than $4 \mathrm{~m}$, with a slightly larger mirror for the Gregorian design than for the Ritchey-Chrétien design. The diameters of the currently largest adaptive mirrors are of the order of $1 \mathrm{~m}$. The manufacture of 4-m-size curved adaptive mirrors is therefore a substantial risk. The situation is slightly more favourable with a Gregorian design since the secondary mirror is concave;

- the focal surface is strongly curved in both designs. The Gregorian design has, in addition, the disadvantage that the focal surface is convex with respect to the exit pupil;

- while for the Gregorian design the secondary mirror is favourably conjugated to a layer a few hundred meters above the ground, it is conjugated to a layer below the ground for the Ritchey-Chrétien design;

- for both designs the images of laser guide stars in the field are strongly aberrated.

Neither of the two designs can therefore fulfil all of the requirements mentioned in Sect. 2.

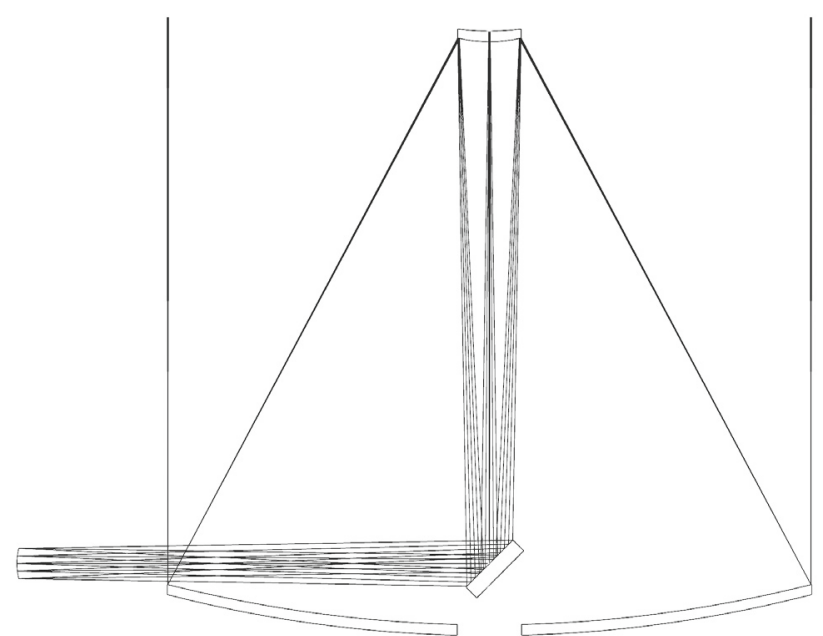

Fig. 1. Cassegrain configuration with a Nasmyth focus.

\subsection{How many mirrors are needed for this telescope?}

To fulfil all of the requirements in Sect. 2, another type of telescope is needed. Starting from a design with two aspheric mirrors like the Ritchey-Chrétien or the Gregorian design, additional features for a telescope with a diameter of the order of $42 \mathrm{~m}$ will increase the required number of mirrors. Only solutions providing a field of view of at least 10 arcmin and having a central obstruction not larger the $10 \%$ of the area will be considered.

The first additional feature, the optional access to a focus position on a Nasmyth platform which is convenient for instrumentation, requires one additional mirror, as shown in Fig. 1.

The second additional feature, the correction of the aberrations generated by atmospheric turbulence in the ground layer by a preferably flat, adaptive $2.5 \mathrm{~m}$ mirror conjugated to a layer a few hundred meters above the ground, requires at least one additional powered mirror that re-images the telescope entrance pupil, usually located at M1, onto the adaptive mirror. This leads to a minimum of four mirrors in the telescope.

The re-imaging of the pupil also generates an intermediate image. A solution with the intermediate focus at the center of M4 is shown in Fig. 2.

A combination of the configurations in Figs. 1 and 2, as shown in Fig. 3, can fulfil all the requirements listed in Sect. 2. The mirror M5 does not only provide access to a convenient focus, but can also be used for field stabilization to relax the requirements on the adaptive mirror $\mathrm{M} 4$, as mentioned in the requirement $\mathrm{A} 4$.

\subsection{Spherical or aspheric primary mirror}

A spherical primary mirror M1 would have strong advantages in terms of cost. Nevertheless, all designs based on spherical primary mirrors require two additional mirrors to achieve the same performance as similar telescopes with aspheric primaries (Sebring \& Ramsey 1997; Stobie et al. 2000; Dierickx \& Gilmozzi 2000). The reason is that the huge spherical aberration introduced by a fast spherical M1 can only be corrected by a mirror conjugated to M1. This conjugation requires two additional mirrors, the first one creating an image of M1 on the second, which corrects most of the spherical aberration. Such a correction of the spherical aberration is only possible if the ratio of the diameter of the correcting mirror to that of M1 is not too large. 


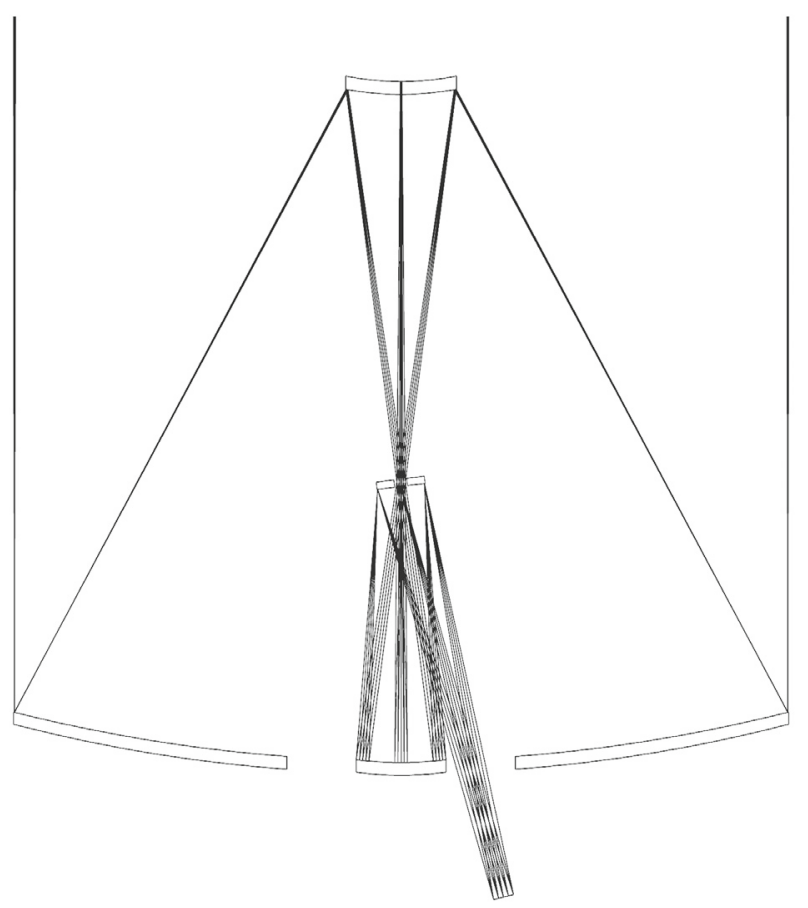

Fig. 2. Optical design with re-imaging of the telescope pupil, located at M1, on to a flat, possibly adaptive mirror.

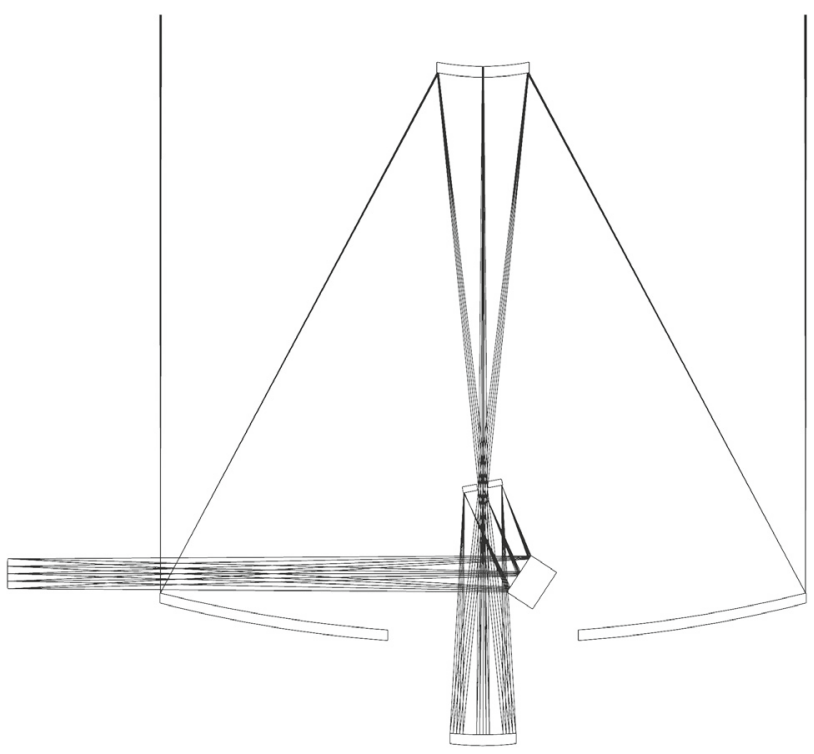

Fig. 3. Optical Layout 1 of the proposed five-mirror design, offering the best compromise between sizes of M2, M4 and M5, the size of the field, and the central obstruction.

For a diameter of M1 of $42 \mathrm{~m}$ the diameter of the correcting mirror will be between 6 and $8 \mathrm{~m}$. A smaller mirror would require an extreme asphericity that would lead to extreme sensitivity of the image quality with respect to misalignments. It would also either strongly reduce the field of view or increase the central obstruction to an unacceptable level.

To comply with the requirements introduced in Sect. 2, 7 mirrors would be required. A possible design is shown in Fig. 4. In an alternative design with one mirror less the two flat mirrors M6 and M7 could be combined in a single mirror located on an image of the pupil to perform the field stabilization. However, due to its $45^{\circ}$ inclination this mirror cannot be used to correct

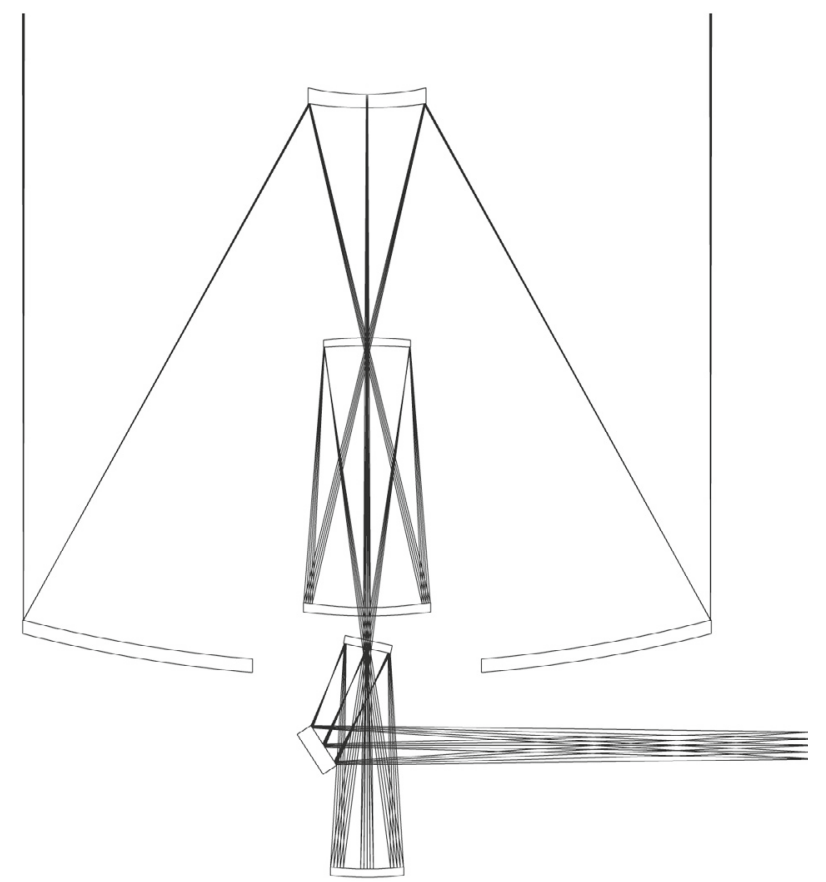

Fig. 4. Optical design for an adaptive telescope with a spherical primary mirror.

aberrations introduced by a specific layer in the atmosphere, since different parts of the mirror are conjugated to different altitudes of the layers. The task of adaptive optics corrections has then to be transferred to M4, which is also on a pupil image. However, due to the size and high asphericity of this mirror, the alternative solution is not attractive.

The only advantages of a spherical mirror are an important cost reduction and an easier alignment, since a rotation of a segment around its normal at the center is irrelevant. These advantages have to be weighed against a long list of negative consequences, some of them resulting from the huge spherical aberration introduced by M1:

- lower throughput and higher emissivity due to two additional mirrors;

- lower image quality over 10 armin field of view;

- strong field curvature in the wrong direction, i.e. opposite to the pupil;

- complex mechanics to support the corrector for the spherical aberration;

- strong aberrations of the image of the laser guide star, which are 10 times larger than in the five-mirror design;

- impossibility to insert an atmospheric dispersion compensator at the intermediate focus since the beam is too fast at the first intermediate focus and the design space available at the second one is too small;

- additional complexity of the wavefront control due to two additional mirrors;

- feasibility of polishing the highly aspheric M4 with a departure of approximately $10 \mathrm{~mm}$ from the best-fitting sphere has not been demonstrated.

As preliminary contacts with optical manufacturers have resulted in positive feedback on the feasibility of an aspheric M1 at an affordable cost (Castro et al. 2000; Nelson \& Mast 1990), it is not obvious that a telescope with a spherical primary mirror will be cheaper - it may turn out to be more expensive. In 
addition, most of the desirable features mentioned above would not be available.

\subsection{The five-mirror concept}

The proposed optical design with five mirrors and an intermediate image originates from an anastigmatic design (Korsch 1972). For the fixed geometry chosen, a restriction to conic surfaces for M1, M2, and M3 limits the achievable image quality over a field with a diameter of 10 arcmin to Strehl ratios of approximately 0.9 for an optimization over the whole field. However, using generalised aspheric coefficients (not a conic section) for M3, the image quality is effectively diffraction limited over the whole field as shown in Table 4. For all layouts presented below, the primary mirror is then elliptical, the secondary hyperbolic, and the third powered mirror a weak general asphere.

M2 and M3 form an intermediate image of the pupil. The two flat mirrors M4 and M5, both having diameters of 2 to $3 \mathrm{~m}$ depending on the layout, are close to the intermediate pupil. M4 is conjugated to a layer $175 \mathrm{~m}$ above the ground and can do the ground layer adaptive optics and low-stroke fast tip-tilt corrections. M5 is sufficiently close to the pupil to do large stroke and comparatively low temporal frequency field stabilization without moving the pupil image in the instruments by unacceptable amounts. M4 and M5 can also be used to correct errors introduced by misaligments and deformations of the first three mirrors. This will, in particular, relax the requirements on the stability of the secondary mirror.

The intermediate image formed by the combination of M1 and M2 has to be located close to the intermediate pupil, in this design close to M4, in order to be inside the central obstruction of the telescope.

The chosen geometry for a given field of view is a compromise between the size of the secondary mirror, the size of the adaptive and field stabilization mirrors, the distance of these mirrors from the pupil, and the position of the elevation axis. On the one hand, a faster beam at the intermediate image reduces the sizes of the intermediate image, the adaptive and the field stabilization mirrors, and the central obstruction of the telescope. On other hand, a faster beam increases the size of the secondary mirror or raises the elevation axis of the telescope.

The two flat mirrors can be mounted on a rotating structure and the optical beam can therefore be directed to either of the two Nasmyth foci.

A large number of similar designs are possible. The two designs labelled Layout 1 and Layout 2, shown in Figs. 3 and 5, respectively, fulfil the following additional requirements:

- the diameter of M2 should not be larger than $6 \mathrm{~m}$;

- the elevation axis should be above M1 to avoid a long light path through the structure. However, it should be close enough to M1 to ease the mechanical balancing of the telescope;

- the diameters of the adaptive and the field stabilization mirrors should not be larger than $2.8 \mathrm{~m}$.

In Layout 2 the third mirror is at the same level as the primary mirror.

Layout 1 offers probably the best compromise between the conflicting requirements mentioned above. Its disadvantage may be the light path through the upper part of the M1 cell structure, which may affect the image quality due to a reduced natural ventilation in this area. Layout 2 is more attractive from this point of view. In addition, the diameter of M2 is slightly smaller and

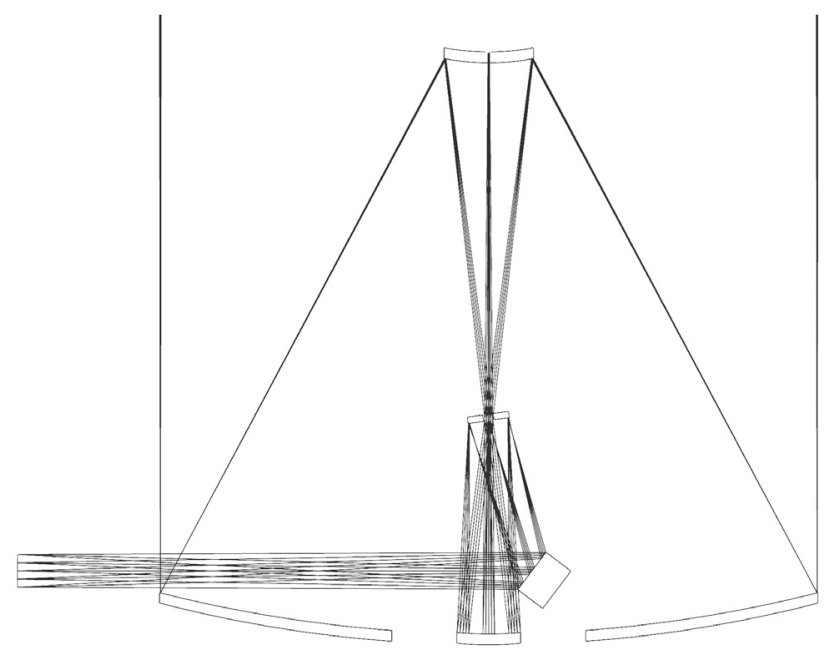

Fig. 5. Optical Layout 2: third mirror at the same level as the primary mirror.

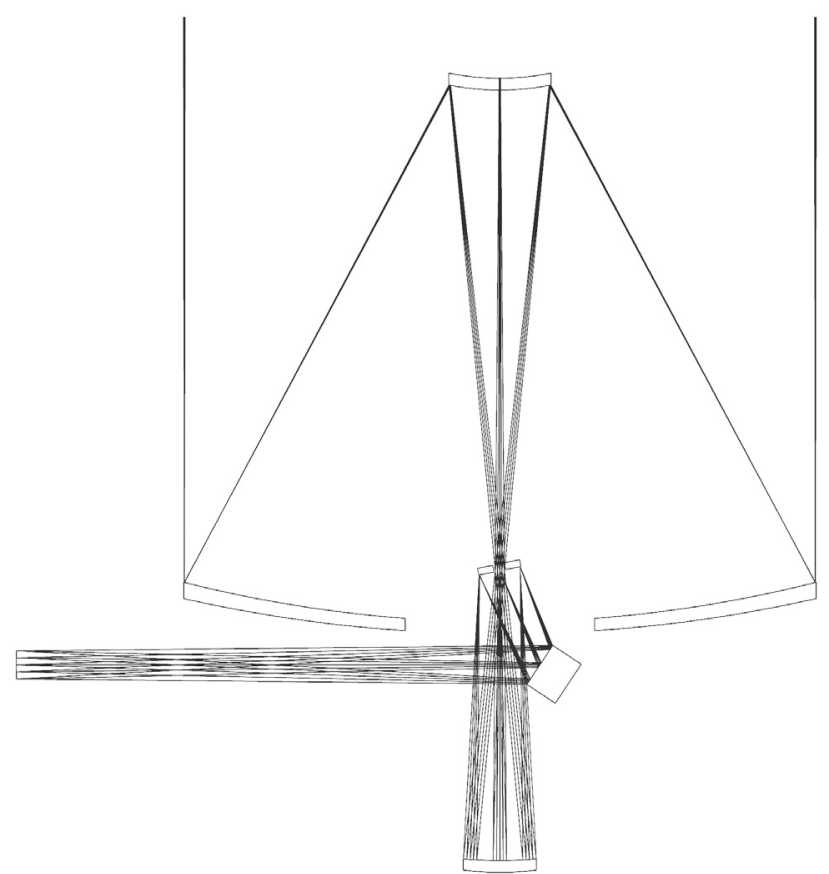

Fig. 6. Optical Layout 3: elevation axis below the primary mirror.

the incidence angle of the beam on M4 is reduced. However, the diameters of M4 and M5 are slightly larger and the distance between them has increased.

In Layout 3, shown in Fig. 6, the elevation axis is below the primary mirror, which makes the balancing of the telescope much easier. However, the consequences would be either a larger diameter of the secondary mirror, which is more than $7 \mathrm{~m}$ for a $42 \mathrm{~m}$ primary mirror, larger diameters of M4 and M5, or a reduction of the field of view. This solution would become more attractive for telescopes with diameters below $35 \mathrm{~m}$.

\subsection{Telescope characteristics}

This section describes only Layout 1 in more detail. However, a similar performance can also be achieved with Layouts 2 and 3. Table 1 shows the fundamental optical design parameters. $T h$ is the distance to the following optical element for the first four mirrors and the distance to the focus for M5, $r_{\text {curv }}$ the radius of 
Table 1. Fundamental optical parameters of the 5-mirror design.

\begin{tabular}{|c|c|c|c|c|}
\hline Surface & $T h / \mathrm{mm}$ & $r_{\text {curv }} / \mathrm{mm}$ & $\begin{array}{l}d_{\text {long }}, d_{\text {short }} \\
\text { outer edge }\end{array}$ & $\begin{array}{l}d_{\text {long }}, d_{\text {short }} \\
\text { inner hole }\end{array}$ \\
\hline M1 & -36200.00 & -84000.0 & 42000 & \\
\hline M2 & 42800.00 & -14800.0 & 5985 & \\
\hline M3 & -16000.00 & -24790.0 & 4290 & \\
\hline M4 & 6000.00 & $\infty$ & 2570,2490 & 710,700 \\
\hline \multirow[t]{6}{*}{ M5 } & -33309.61 & $\infty$ & 2834,2360 & 350,200 \\
\hline & Surface & $\begin{array}{r}\text { Conic } \\
\text { constant }\end{array}$ & $\begin{array}{l}\text { Aspheric } \\
\text { coefficients }\end{array}$ & \\
\hline & M1 & -0.992726 & & \\
\hline & M2 & -2.307544 & & \\
\hline & M3 & & $.24685 \times 10^{-15}$ & \\
\hline & & & $.80083 \times 10^{-24}$ & \\
\hline
\end{tabular}

Table 2. Derived first-order parameters of the five-mirror design.

\begin{tabular}{lc}
\hline \hline Effective focal length & $672 \mathrm{~m}$ \\
Final focal ratio & 16 \\
Image scale & $1 \mathrm{arcsec} \equiv 3.25 \mathrm{~mm}$ \\
Angular field of view & $10 \mathrm{arcmin}$ \\
Linear field of view & $1.95 \mathrm{~m}$ \\
Focal ratio of M1 & 1.0 \\
Focal ratio at the intermediate focus & 4.6 \\
Radius of field curvature & $-36 \mathrm{~m}$ \\
Position of the exit pupil & $-36 \mathrm{~m}$ \\
\hline
\end{tabular}

Table 3. Sensitivities of wavefront errors and lateral and longitudinal shift of the image to rigid body movements of M2 and M3.

\begin{tabular}{cc|ccc}
\hline \hline & & $\Delta P_{\text {lat }}$ & $\Delta \varphi$ & $\Delta P_{\text {long }}$ \\
& & $1 \mathrm{~mm}$ & $10 \operatorname{arcsec}$ & $1 \mathrm{~mm}$ \\
\hline & $\sigma_{\mathrm{w}} / \mathrm{nm}$ & 3389 & 1062 & \\
M2 & $\Delta F_{\text {lat }} / \mathrm{mm}$ & 12.6 & 9.0 & \\
& $\Delta F_{\text {long }} / \mathrm{mm}$ & & & 254 \\
\hline \multirow{3}{*}{ M3 } & $\sigma_{\mathrm{w}} / \mathrm{nm}$ & 48 & 27 & \\
& $\Delta F_{\mathrm{y}} / \mathrm{mm}$ & 5.3 & 5.3 & \\
& $\Delta F_{\mathrm{z}} / \mathrm{mm}$ & & & 13 \\
\hline
\end{tabular}

curvature, $d_{\text {long }}$ and $d_{\text {short }}$ are the long and short diameters of the mirrors M4 and M5 with an elliptical shape of the outer edge and the inner hole. For all other mirrors $d_{\text {long }}=d_{\text {short }}$. M4 is tilted by $11.5^{\circ}$ with respect to the on-axis ray, and M5 by $33.5^{\circ}$.

The first order characteristics derived from the fundamental optical design parameters are listed in Table 2. The position of the exit pupil is given with respect to the Nasmyth focus. Table 3 shows the sensitivity of the rms $\sigma_{\mathrm{w}}$ of the wavefront error, the lateral pointing error $\Delta F_{\text {lat }}$ in the focal plane, and the longitudinal defocus $\Delta F_{\text {long }}$ to rigid body movements of M2 and M3, namely a lateral decentering $\Delta P_{\text {lat }}$ of $1 \mathrm{~mm}$, a longitudinal decentering $\Delta P_{\text {long }}$ along the optical axis of $1 \mathrm{~mm}$, and a tilt $\Delta \varphi$ around one of the axes perpendicular to the optical axis of 10 arcsec.

\subsection{Image quality}

For geometrical reasons the field is limited to 10 arcmin, since a larger field would require a faster primary mirror, a larger M2 or a larger M4 combined with a larger M5. The spot diagrams in Fig. 7 illustrate the perfect image quality provided by the fivemirror design. Table 4 shows the wavefront errors and the Strehl ratios at wavelength of $\lambda=450 \mathrm{~nm}$ and $\lambda=2000 \mathrm{~nm}$. The telescope is therefore effectively diffraction limited over the whole field of view even for blue light. Figure 8 shows that the central

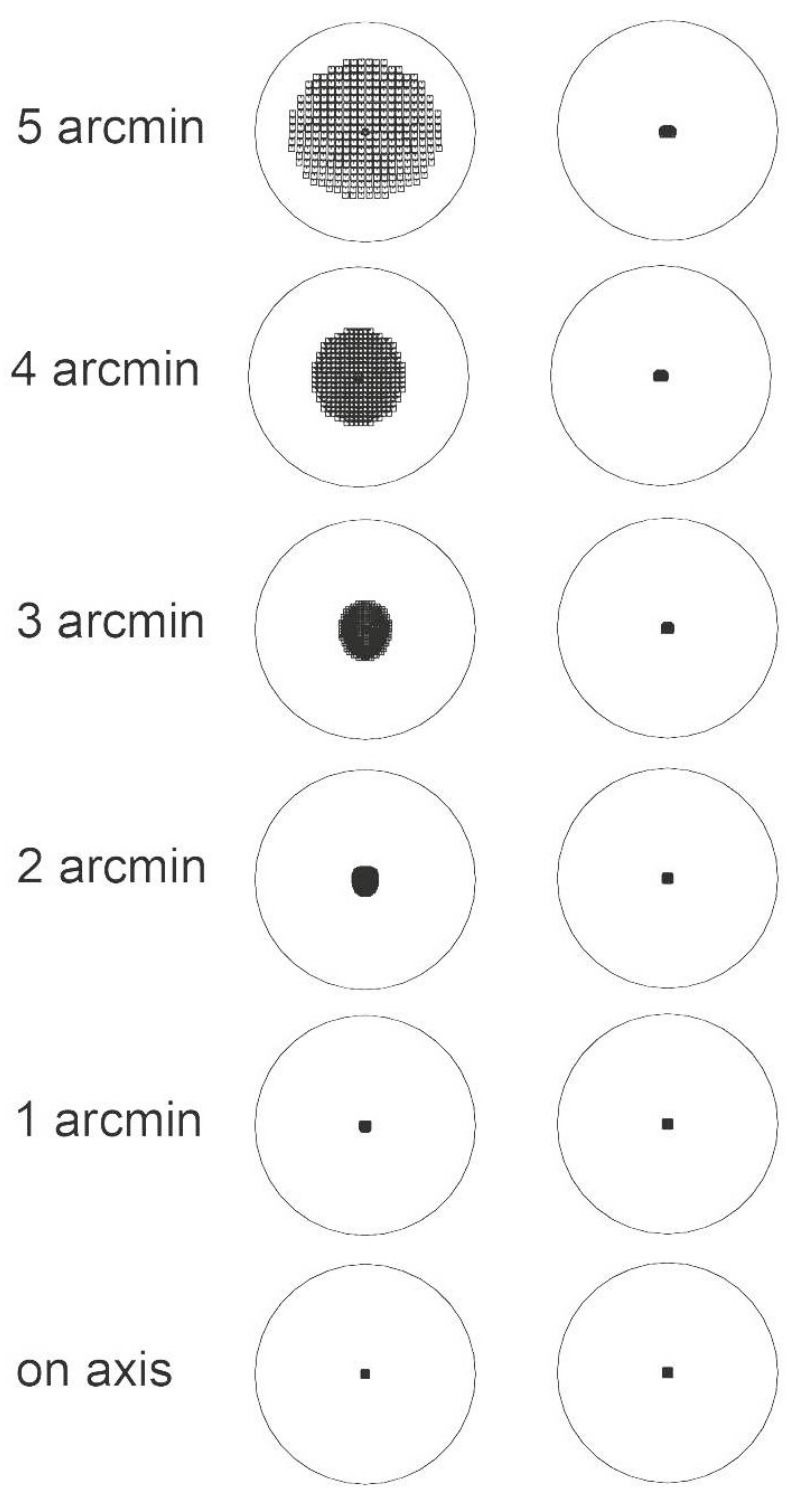

Fig. 7. Spot diagrams at various field angles (radii) for images produced by a Ritchey-Chrétien (left) and the five-mirror design (right) with identical f-numbers of the primary mirrors and the telescopes. The diameter of the circles is 0.2 arcsec.

Table 4. Rms of the wavefront error and Strehl ratios for wavelength of $\lambda=450 \mathrm{~nm}$ and $\lambda=2000 \mathrm{~nm}$.

\begin{tabular}{cccc}
\hline \hline $\begin{array}{c}\text { Field radius } \\
\text { / arcmin }\end{array}$ & $\sigma_{\mathrm{w}} / \mathrm{nm}$ & $\begin{array}{c}r_{\mathrm{s}} \\
\text { at } \lambda=450 \mathrm{~nm}\end{array}$ & at $\lambda=2000 \mathrm{~nm}$ \\
\hline on axis & 8 & 0.99 & 1.00 \\
1 & 8 & 0.99 & 1.00 \\
2 & 8 & 0.99 & 1.00 \\
3 & 10 & 0.99 & 1.00 \\
4 & 11 & 0.98 & 1.00 \\
5 & 15 & 0.96 & 1.00 \\
\hline
\end{tabular}

obstruction of the telescope is defined by the diameter of the hole in the adaptive mirror that is required to let a 10 arcmin field at the intermediate focus pass through. This obstruction, which is $9 \%$ of the area, is much larger than the one of $1 \%$ of the area that would be produced by the shadow of the secondary mirror and would be typical for a Ritchey-Chrétien or a Gregorian design. 


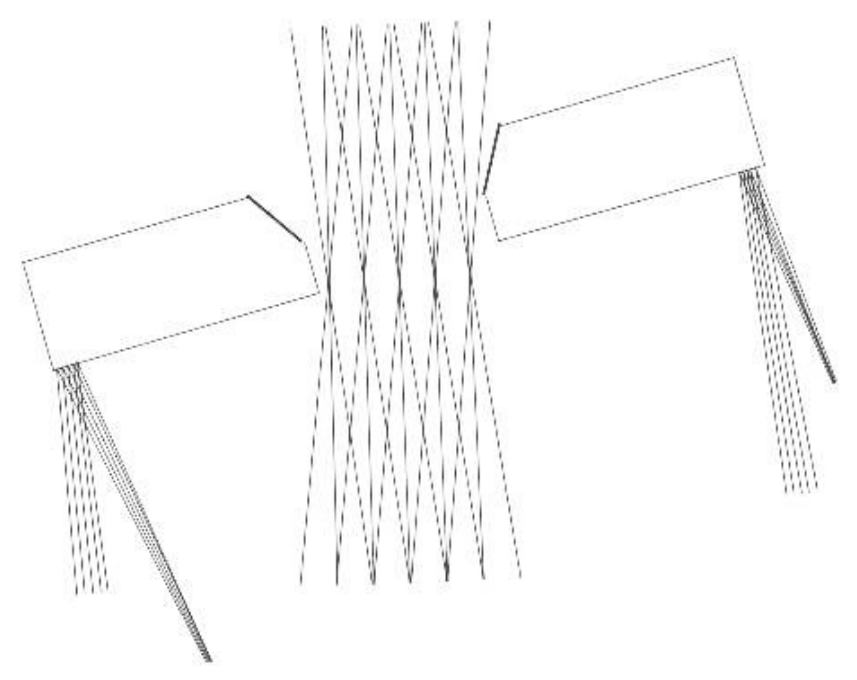

Fig. 8. Size of the hole in M4 that is required to allow a field of view with a diameter of $10 \mathrm{arcmin}$, and that defines the central obstruction.

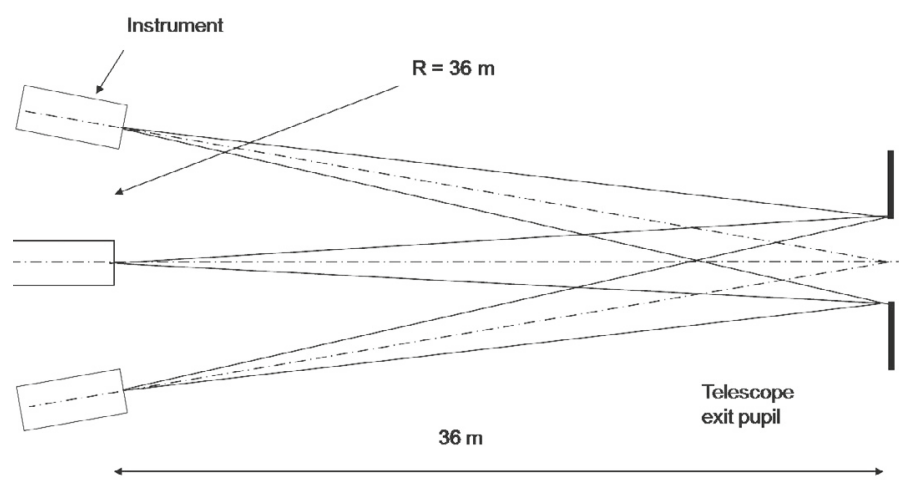

Fig. 9. The radius of the field curvature is identical to the distance between the telescope exit pupil and the focal surface.

Within a certain range, the geometry of the system allows for control of the field curvature. The basic optical parameters can be adjusted such that the field curvature is concentric to the exit pupil of the telescope, as shown in Fig. 9, with a radius of the field curvature of $36 \mathrm{~m}$. This is ideal for the instruments since each point of the field of view will be perpendicular to the beam and will have the same optical quality. The same instrument can therefore be located everywhere in the field of view without any impact on its performance. No correctors are needed as would be the case for a Ritchey-Chrétien or a Gregorian telescope.

To achieve an image quality as close as possible to the theoretical values all powered mirrors should be active.This will allow, including corrections done by M4, the suppression of all low spatial frequency aberrations. The final image quality due to the telescope optics will then be limited by high spatial frequency polishing errors. A complete error budget is not in the scope of this paper.

\subsection{Adaptive optics}

The adaptive mirror M4 is a technologically challenging optical device (Riccardi et al. 2002, 2003) and should therefore be as small as possible. However, its minimum size is determined by the required capability of the telescope to deliver a significant improvement of the seeing over a large field of view. This, in turn, depends on its capability to correct comparatively
Table 5. Rms of the wavefront error of laser guide star sources at various heights and field positions.

\begin{tabular}{ccccc}
\hline \hline $\begin{array}{c}\text { Layer } \\
\text { altitude } / \mathrm{km}\end{array}$ & on axis & $2 \operatorname{arcmin}$ & 3 arcmin & 4 arcmin \\
\hline 180 & 22 & 28 & 45 & 73 \\
150 & 25 & 33 & 54 & 88 \\
120 & 30 & 41 & 67 & 111 \\
90 & 38 & 54 & 91 & 149 \\
\hline
\end{tabular}

low-order aberrations generated, on the one hand, by atmospheric turbulence in the ground layer and, on the other hand, by the telescope itself due to its exposure to the wind. In the current baseline of the design of the $42 \mathrm{~m}$ E-ELT telescope, the adaptive mirror has approximately 5000 actuators with strokes of $100 \mu \mathrm{m}$. To achieve such large strokes requires, with the currently available technology, shells with thicknesses of the order of $2 \mathrm{~mm}$ and distances between the actuators of the order of at least $25 \mathrm{~mm}$. The minimum size of M4 would then be of the order of $2 \mathrm{~m}$. In the current optical design the flat M4 of elliptical shape has lengths of the main axes of $2570 \mathrm{~mm}$ and $2490 \mathrm{~mm}$ and is tilted with respect to the optical axes by $11.5^{\circ}$.

Apart from the large strokes achievable with a rather large adaptive mirror in the telescope, the other advantage is that the effects of ground-layer turbulence can be corrected over a large field of view. Post-focal adaptive optics are limited to a field of view of approximately 2 arcmin. For some modes of observation like multi-conjugate or extreme adaptive optics, a post-focal deformable mirror with much smaller strokes than the adaptive M4 will be added.

M5 is a flat mirror of elliptical shape with lengths of the main axes of $2834 \mathrm{~mm}$ and $2360 \mathrm{~mm}$. It is tilted by $33.5^{\circ}$ with respect to the telescope axis and feeds the Nasmyth focal plane. This mirror has to do the low-frequency, high-stroke field stabilization, that is, to correct pointing errors that have not been corrected by the drives of the main axes. Such residual errors are due to wind load and low temporal frequency atmospheric tip-tilt and may have amplitudes up to 1 arcsec on the sky.

The excellent optical quality of the telescope over a large field of view is also reflected in the quality of the images of the laser guide stars, as shown in Table 5. Classical telescopes like a Ritchey-Chrétien or a Gregorian will introduce aberrations 10 times as high.

Due to the long focal length of the telescope, the laser guide star images are, however, strongly defocused as shown in Fig. 10, and the analysis of the wavefront originating from the laser guide stars can therefore probably not be done in the same adaptor as the analysis of the wavefront originating from natural guide stars.

\subsection{Full-field atmospheric dispersion compensator}

A full-field atmospheric dispersion corrector near the Nasmyth focus would require very large optical components with diameters of more than $2 \mathrm{~m}$. No glasses of these sizes are available. The lenses or prisms would have to be mosaics. Linear atmospheric dispersion correctors cannot be used due to the displacement and tilt of the focal plane they introduce. For the following reasons, the intermediate focus is a better location for a full-field atmospheric dispersion corrector:

- only one atmospheric dispersion corrector is needed for all foci;

- optical components are smaller and available; 


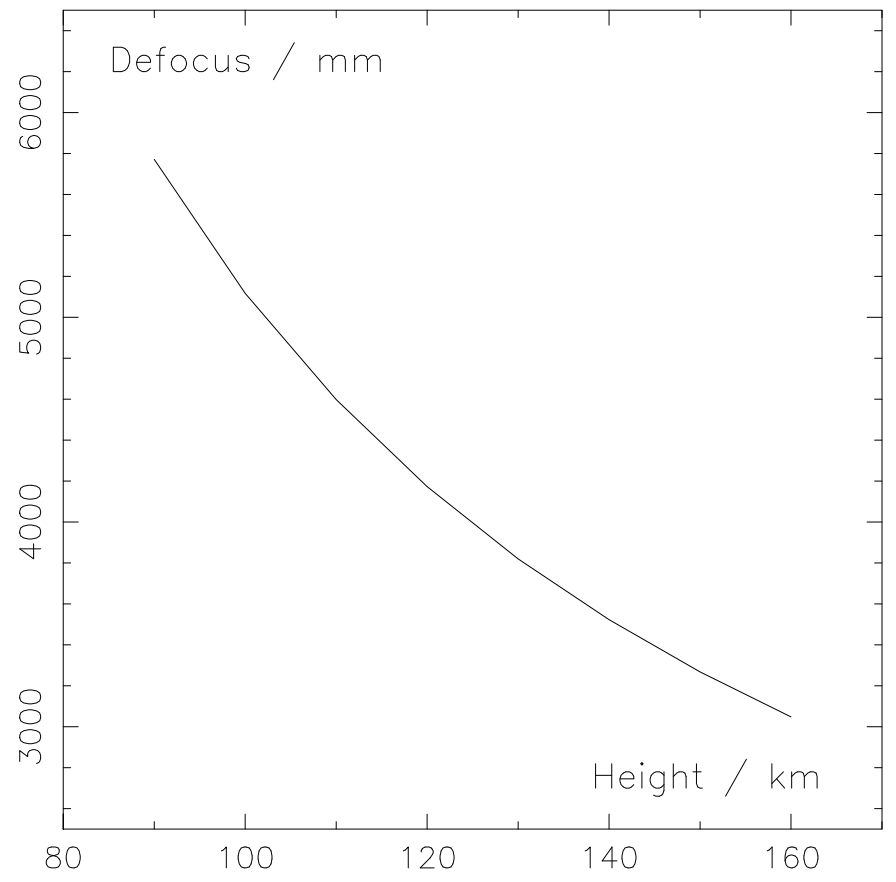

Fig. 10. Defocus as a function of the height of the laser guide star.

- a linear atmospheric dispersion corrector can be used because focal plane displacements and tilts can be compensated by the mirrors further down in the optical train;

- prism angles are smaller due to the faster focal ratio at the intermediate focus.

The atmospheric dispersion corrector mainly introduces defocus, astigmatism, and smaller amounts of spherical aberration and coma. These aberrations can be corrected in the following way:

- defocus by moving M2 along the optical axis;

- pointing errors by an offset of the telescope pointing;

- coma by a lateral decentering of M2;

- Astigmatism and spherical aberration by a deformation of $\mathrm{M} 2$.

Astigmatism and spherical aberration could also be corrected by M4, but this would reduce the stroke available for these mirrors to correct the effects of atmospheric turbulence.

For observations over small fields of view, this atmospheric dispersion corrector would have to be removed from the beam and the correction done near or inside the instrument. Since, in that case, small prisms are sufficient, the large choice of available materials will permit a much better correction.

\subsection{Focus positions}

\subsubsection{Nasmyth and gravity stable foci}

The telescope focal plane can be shifted to any location on the Nasmyth platform by movements of M2 and M3 along the optical axis. The nominal optical quality can easily be recovered by appropriate deformations of these active mirrors.

As shown in Fig. 13, a sixth mirror can be inserted in front of a Nasmyth focus to provide a gravity stable focus with a vertical rotation axis that could be required for very heavy and complex instruments.

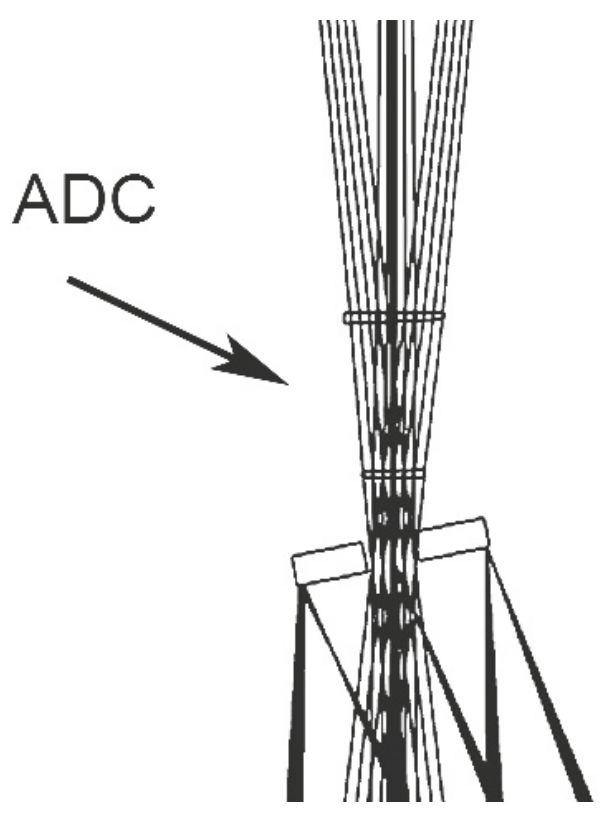

Fig. 11. Location of the atmospheric dispersion corrector in front of the intermediate focus.

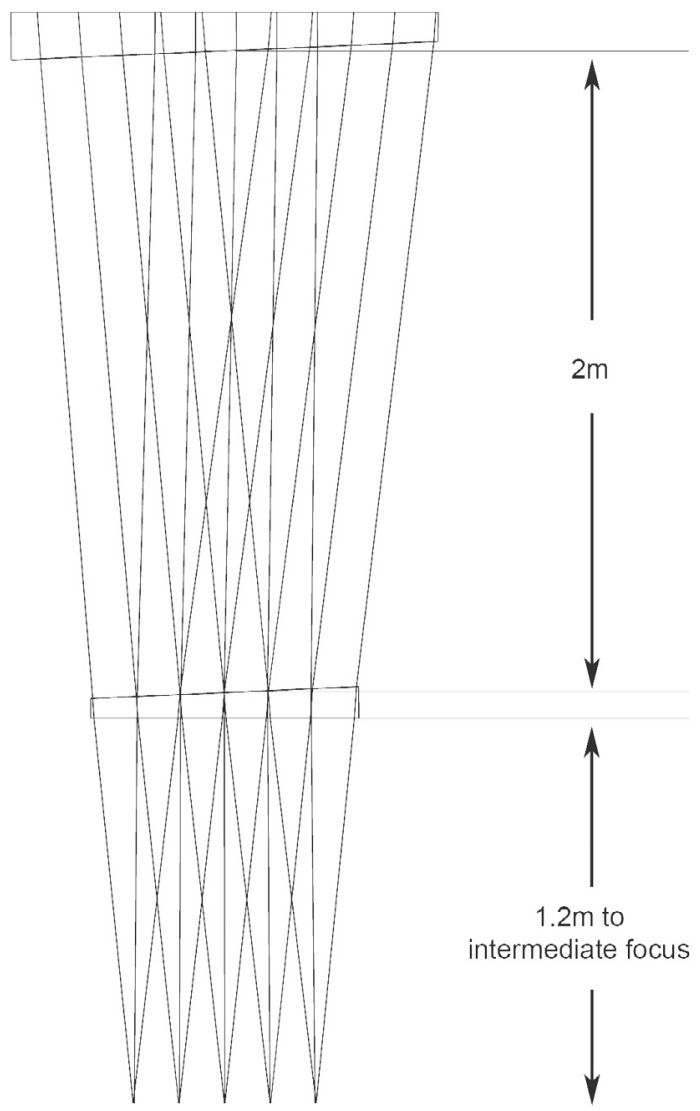

Fig. 12. Optical design of the atmospheric dispersion corrector.

\subsubsection{Coudé focus}

A coudé focus is needed for a very high resolution spectrograph that needs to be located in a very stable environment. There are several possible concepts for the coudé focus, depending on the field of view. The maximum field that can be transferred to the coudé focus is 5 arcmin, but the relay optics would require 3 large flat mirrors of elliptical shape with diameters of 


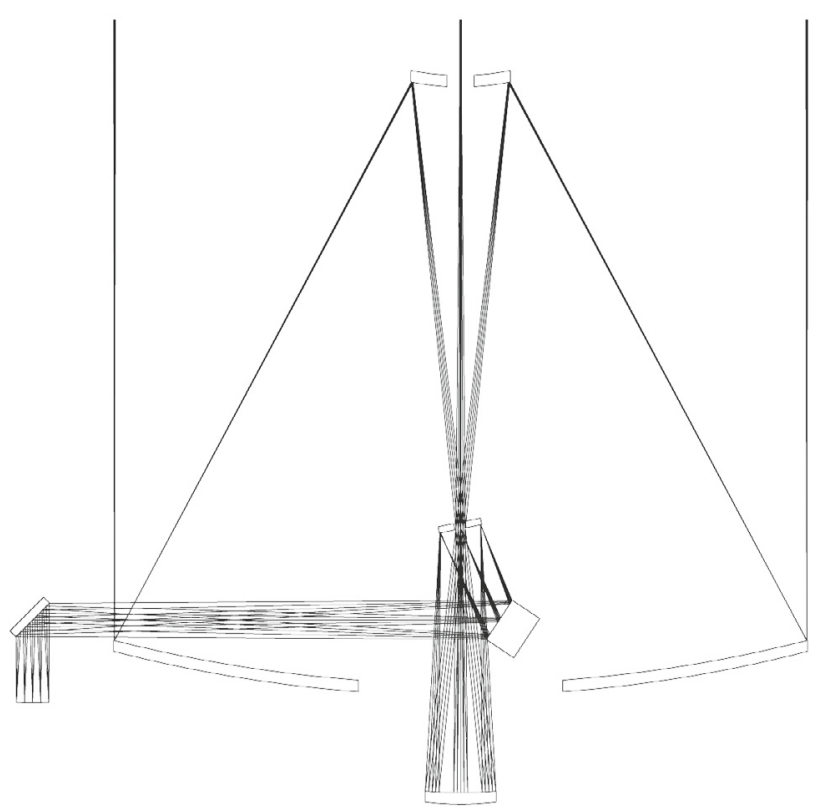

Fig. 13. Gravity-stable focus generated by a folding flat mirror.

approximately 2 and $3 \mathrm{~m}$ along the main axes. The transfer of the focal plane to the coudé focus (is) would be achieved by axial translations of M2 and M3 and a modification of their asphericities. However, since observations at infrared wavelengths are not foreseen at this focus, a more economical solution shown in Fig. 14 with smaller optics consisting of two doublets and two folding flats, would be to transfer a 20 arcsec field of view sufficient for a very high resolution spectrograph. Spot diagrams showing the expected image quality for this solution are shown in Fig. 15. For this design, the image quality is limited by chromatism that cannot be fully corrected since the required abnormal dispersion glasses are not available at the large size of one of the two doublets.

The two lenses have diameters of 340 and $680 \mathrm{~mm}$, respectively. The wavelength range of the coudé focus is limited to that of the high-resolution spectrograph, that is to the range from $450 \mathrm{~nm}$ to $900 \mathrm{~nm}$.

The coudé focus will require only one wavefront sensor for field stabilization. All other functions of the telescope can still be controlled by wavefront sensors at the Nasmyth focus.

\subsubsection{Intermediate focus}

The five-mirror design also offers an intermediate focus that could be used for small instruments, for example for observations at thermal infrared wavelengths. The optical quality of the telescope at this focus is not as good as the optical quality at the other foci. Using M2 to correct for defocus and spherical aberration, a seeing-limited optical quality of 0.2 arcsec full-widthat-half-maximum can be achieved over a field of 1 arcmin. Field coma is the limiting aberration. Although this focus may appear an attractive feature, it is not obvious how the telescope can be controlled in this configuration, since the correction capabilities of M4 and M5, in particular for field stabilization, are not available at this focus.

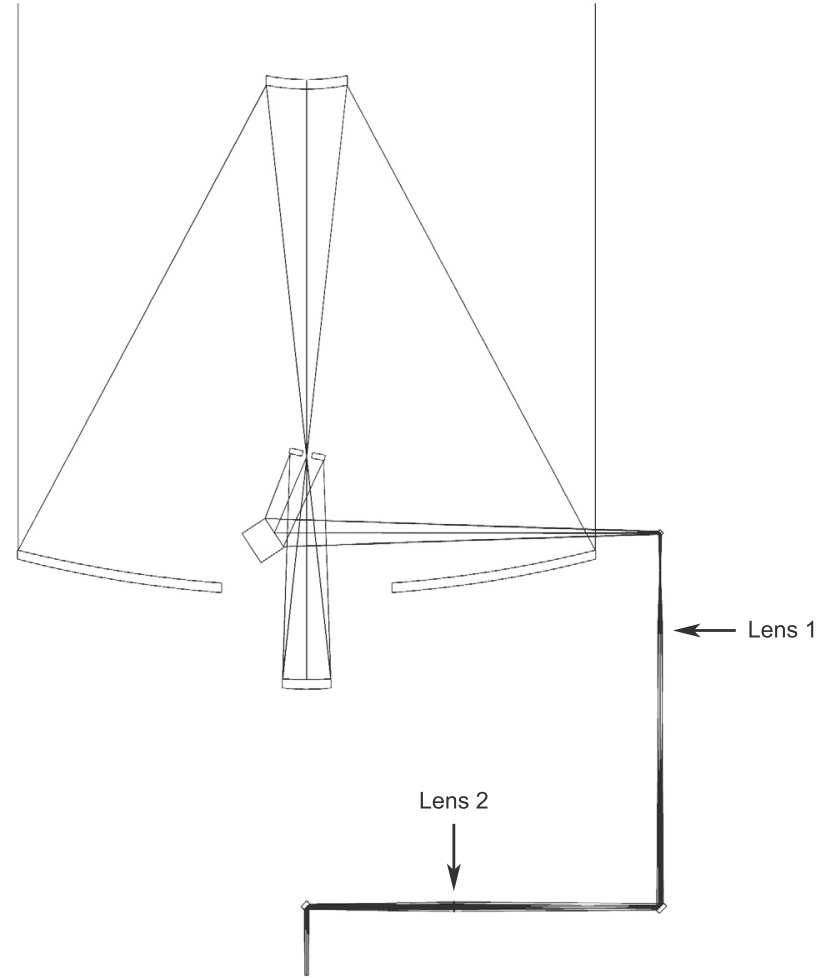

Fig. 14. Optical layout of the coudé configuration.

\section{$10 \operatorname{arcsec}$}

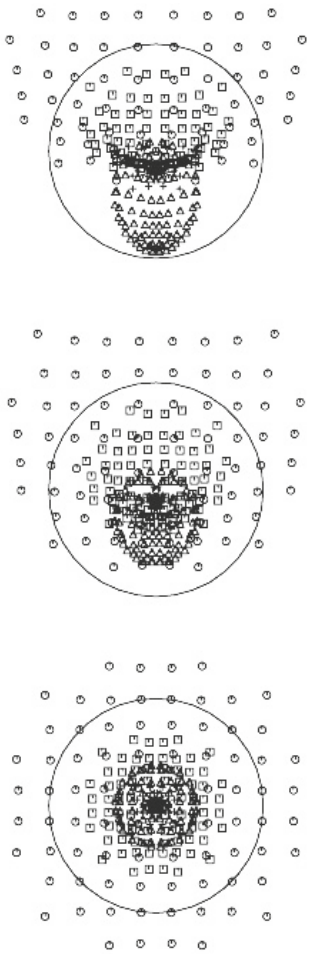

Fig. 15. Optical quality at the coudé focus. The diameter of the circle is $0.1 \mathrm{arcsec}$.

\section{Conclusions}

Since November 2006 the presented five-mirror design for the optics of an adaptive extremely large telescope is the baseline for the European Extremely Large Telescope. Its major advantages are the effectively diffraction limited image quality even at visible wavelength over a field of view of 10 arcmin, the instrument 
friendliness due the focal surface being concentric to the exit pupil, and the excellent quality of the images of the spots generated by the laser guide stars.

Acknowledgements. The author would like to thank L. Noethe and R.N. Wilson for helpful suggestions for the manuscript and the referee for useful comments.

\section{References}

Castro, J., Devaney, N., Jochum, L., Ronquillo, B., \& Cavaller, L. 2000, Proc. SPIE, 4003, 24

Dierickx, Ph., \& Gilmozzi, R. 2000, Proc. SPIE, 4004, 290
Gilmozzi, R., \& Spyromilio, J. 2007, ESO Messenger, 127

Korsch, D. 1972, Appl. Opt., 11, 2986

Nelson, J. E., \& Mast, T. S. 1990, Proc. SPIE, 1236, 47

Riccardi, A., et al. 2002, ESO Conf. and Workshop Proc., Beyond Conventional Adaptive Optics, Venice, 58, 55

Riccardi, A., Brusa, G., Salinari, P., et al. 2003, Proc. SPIE, 4839, 721

Sebring, T. A., \& Ramsey, L. W. 1997, Proc. SPIE, 2871, 32

Stobie, R., Meiring, J. G., \& Buckley, D. H. 2000, Proc. SPIE, 4003, 355

Wilson, R. N. 1998, Principles of Adaptive Optics I, 2nd edn. (New York: Academic Press)

Wilson, R. N. 2004, Reflecting Telescope Optics I, 2nd edn. (Berlin: Springer) 